Archives

9| 1992

Varia

\title{
Le bois, le fer et l'eau en foret d'Othe à la fin du Moyen Âge : bilan et perspectives
}

Patrice Beck, Philippe Braunstein et Michel Philippe

\section{(2) OpenEdition}

\section{Journals}

Édition électronique

URL : http://journals.openedition.org/ccrh/2794

DOI : $10.4000 /$ ccrh. 2794

ISSN : 1760-7906

Éditeur

Centre de recherches historiques - EHESS

Édition imprimée

Date de publication : 15 avril 1992

ISSN : 0990-9141

Référence électronique

Patrice Beck, Philippe Braunstein et Michel Philippe, « Le bois, le fer et l'eau en foret d'Othe à la fin du Moyen Âge : bilan et perspectives », Les Cahiers du Centre de Recherches Historiques [En ligne], 9| 1992, mis en ligne le 18 mars 2009, consulté le 19 avril 2019. URL : http://journals.openedition.org/ ccrh/2794; DOI : 10.4000/ccrh.2794

Ce document a été généré automatiquement le 19 avril 2019

Article L.111-1 du Code de la propriété intellectuelle. 


\title{
Le bois, le fer et l'eau en foret d'Othe à la fin $\mathrm{du}$ Moyen Âge : bilan et perspectives
}

\author{
Patrice Beck, Philippe Braunstein et Michel Philippe
}

1 Pour rendre à l'industrie ancienne sa véritable dimension dans des sociétés où, faute de sources explicites, on ne voulait connaitre que des artisans, il faut prospecter les zones forestières et le cours des rivières où les traces fossiles de la métallurgie permettent d'imaginer le potentiel et la mise en œuvre des ressources naturelles.

2 Assurément, les sources écrites ne sont pas, avant l'époque moderne, à la hauteur du phénomène, et les médiévistes ont pris leur parti d'utiliser des sources obliques et, sans doute, déformantes: confirmations et donations de terres et de revenus à des établissements ecclésiastiques, arbitrages et jugements en cas de conflits entre utilisateurs de l'eau et des bois. Seules des comptabilités permettraient de saisir l'activité et les résultats d'entreprises qui, indéniablement, étaient ouvertes sur le marché. Quant aux études de sites, elles nourrissent des hypothèses sur la capacité de travail des installations, et il est évident que si l'entreprise minière et métallurgique a durablement imprimé sa marque sur le paysage, la datation des vestiges est une impérieuse nécessité pour toute évaluation d'un niveau de production.

Dans le cadre ainsi tracé pour une double approche du phénomène industriel par les textes et le terrain, la forêt d'Othe, aux confins de la Champagne et de la Bourgogne, offrait un champ privilégié. Ne faisaient défaut ni les travaux anciens, ni les promenades archéologiques ; mais les sources, publiées ou inédites, manquaient d'un projet global qui les anime ${ }^{1}$. Le branle fut donné par l'analyse approfondie d'une série de comptes d'exploitation de trois grosses forges créées par la comtesse de Flandre en 1372 dans sa châtellenie de Champagne: la comptabilité de l'entreprise en régie directe saisissait le cycle productif, de l'extraction du minerai à la vente du fer « marchand», et éclairait les rapports sociaux entre paysans, exploitants des forges et autres fermiers des droits comtaux, administrateurs et marchands de métal ${ }^{2}$.

4 Une enquête systématique ayant été décidée, ses premières étapes étendirent le champ couvert par la documentation écrite, révélant en particulier une série de comptes 
d'exploitation d'autres forges dans la seigneurie voisine des évêques de Troyes, et déployant le panorama à d'autres secteurs de la forêt et d'autres époques de l'exploitation minière. De premiers sondages sur des sites explorés révélaient, par chance, que l'arc chronologique s'étendait dans la très longue durée, de l'âge du Fer à l'extrême fin du Moyen Âge ${ }^{3}$. Au moment où se terminent pour l'essentiel l'enquête documentaire et une première phase de prospection, il a semblé utile et juste de présenter un bilan intermédiaire, en insistant sur les acquis et les enjeux ${ }^{4}$.

5 La prospection s'est largement déployée sur le terrain, privilégiant les massifs boisés systématiquement explorés, mais s'étendant aussi, en fonction des données archivistiques, cartographiques et orales rassemblées, aux champs et aux fonds de vallées. Plus d'un millier d'hectares ont été soigneusement visités; 140 sites, essentiellement forestiers et souvent complexes, ont été repérés autour d'Aix-en-Othe, de Bœurs-en-Othe, de Dilo, de Rigny-le-Ferron et de Pâlis. Une soixantaine de sites d'extraction ont été reconnus, dont plus de la moitié sont très probablement des minières en raison de la présence de minerai et de ferriers associés aux fosses et bombements caractérisant la topographie des lieux. Plus d'une centaine de sites à scories, c'est-à-dire, à dechets de réduction, dont les trois quarts sont des ferriers conservés en place, plus une douzaine, des ferriers probables mais arasés, sont désormais enregistrés.

Le développement de ces ferriers est, en général, mesuré, d'au plus quelques milliers de tonnes, d'après les évaluations de cubage ${ }^{5}$. Le plus important, situé au-dessus de Bérulle, d'environ $300 \mathrm{~m}$. de long, 20 à $30 \mathrm{~m}$. de large et d'une hauteur, en son centre, de l'ordre de $3 \mathrm{~m}$., doit totaliser plus de 12.000 tonnes de masse. Deux autres se distinguent encore, mais loin derrière, en présentant des masses de 7000 et 5600 tonnes; six oscillent entre 1000 et 3700 tonnes et trois, au-dessus de 700 tonnes, mais les suivants n'en n'ont pas 500 , et certains sont même très petits, de quelques mètres d'extension, de moins de 10 tonnes de masse.

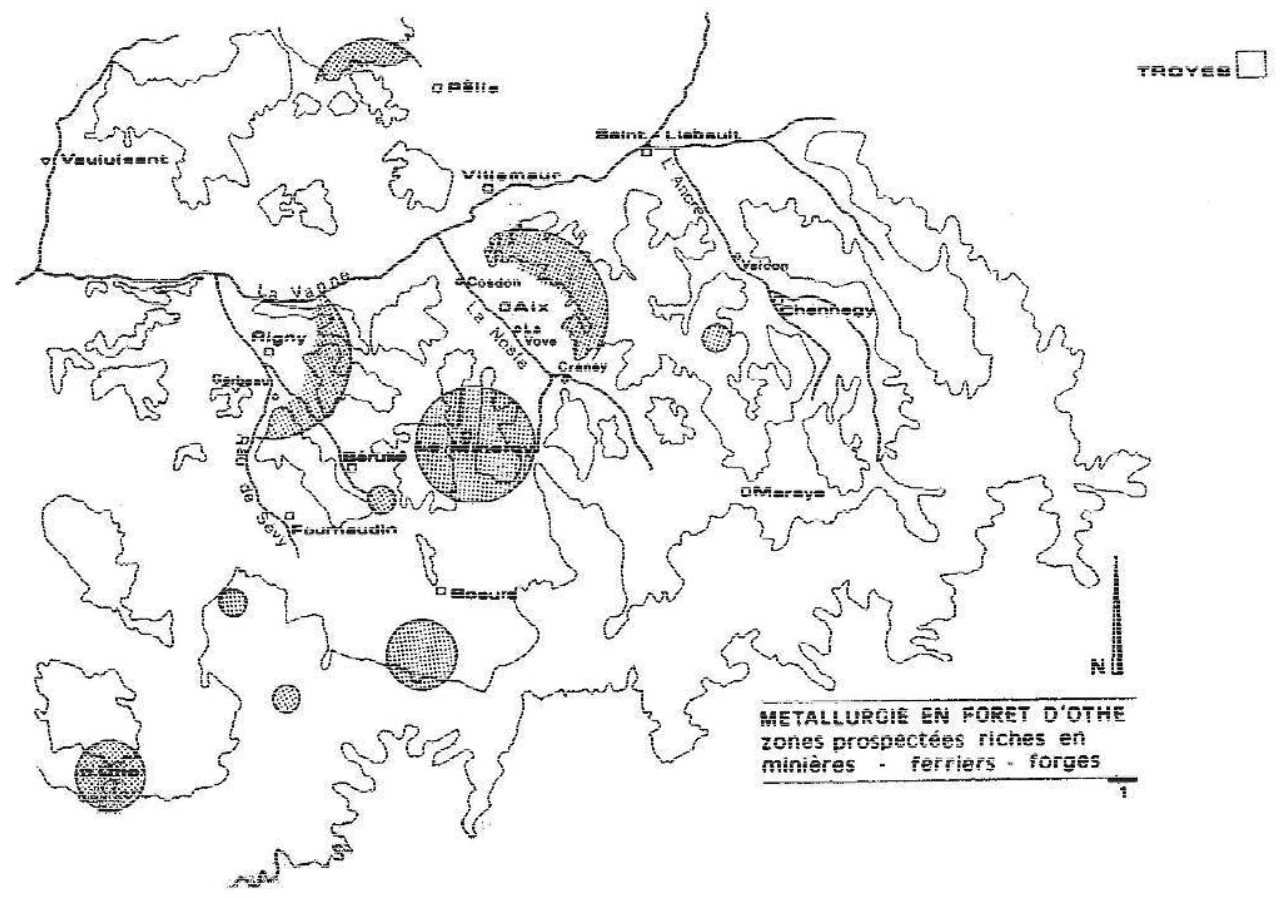

7 Certains ferriers paraissent isolés, mais ce peut être l'effet d'une prospection malchanceuse, perdus de surcroît au centre d'épais massifs boisés, telles les vastes forêts 
des Rajeuses et de Venizy, qui séparent les clairières d'Arces-Dilo et de Bœurs-en-Othe. Mais la plupart, sans être véritablement regroupés, s'égrènent en chapelets plus ou moins lâches, à proximité des lisières actuelles, dans des cantons de bois irrigués par des réseaux de chemins conduisant à des habitats constitués aujourd'hui de hameaux, de fermes ou d'anciens établissements industriels sur l'eau. Partout la toponymie et la micro-toponymie renvoient à l'activité minière et métallurgique.

Ainsi, au-dessus d'Aix-en-Othe, les sites d'extraction et de réduction s'alignent à la lisière de la forêt communale bordant le bois des Brosses. L'une des parcelles se nomme La Forge et, séparant les champs du Fourneau et de la Forge de Fau, le chemin du Fourneau conduit tout droit vers le hameau de la Vove qui, sur la Nosle, a conservé son moulin. Sur l'autre versant de la vallée et dominant le même site, où l'énergie hydraulique anima à la fin du Moyen Age et au début de l'époque moderne divers établissements industriels, le bois de la Rachée et les champs gagnés sur lui, comme le suggère le nom de l'un d'entre eux, Buisson Brûlé, ont livré un ferrier et des nodules de grès ferrugineux en abondance.

Prenant appui sur les indices puissants que représentent les ferriers, dans une topographie souvent tourmentée, la prospection a été particulièrement attentive à l'enregistrement des données, dans la perspective d'un traitement ultérieur approfondi. Il s'agissait d'abord du prélèvement d'échantillons de scories et, si possible, de minerai. Les sites à scories livrent dans l'ensemble des éléments lourds, chargés en fer, mais aussi des amas de scories bulleuses, spongieuses, vitrifiées; si le procédé direct caractérise la sidérurgie de la forêt d'Othe comme celle des pays voisins, des documents d'archives ont révélé la précoce introduction du procédé indirect : la présence de laitiers foncés, vitreux, homogènes à la partie supérieure d'un ferrier surplombant un cours d'eau, dont la retenue est toujours visible, pourrait attester cette mutation technique.

D'autres données ne sont pas moins essentielles pour aider à recomposer un paysage industriel disparu : indices topographiques et phytologiques d'équipements hydrauliques, tracés d'anciens chemins, zones de travail, qu'il s'agisse de carrières, dont différents horizons ont été exploités pour des usages locaux (silex, marnes, calcaires à chaux), de charbonnières, de bases de cabanes.

11 Comment ordonner chronologiquement le foisonnement des situations rencontrées sur le terrain, établir les phases d'une activité sidérurgique échelonnée sur des millénaires, établir des corrélations certaines entre le terrain et les sources écrites? Plus précisément, comment concentrer l'enquête sur les seuls sites dont on puisse confronter la structure avec les informations tirées des archives?

12 Avant d'en venir à l'apport des sources écrites - en soi, capital -à l'histoire de la sidérurgie champenoise à la fin du Moyen Age, redisons brièvement que l'espoir de conjuguer les deux approches ne paraît pas vain à ce stade de l'enquête, puisque de fortes présomptions s'accumulent pour identifier l'un des ferriers qui fut l'objet d'un sondage en 1989 avec l'un de ceux que décrivent les témoins d'un procès entre l'abbé de Pontigny et l'évêque de Troyes. Il n'en n'est pas moins vrai que, mis sur la piste des ferriers de Pâlis par les comptes de gestion des forges à la fin du XIV e siècle, nous nous sommes retrouvés sur le terrain à l'âge du Fer. Il faut donc se garder de juxtaposer sur une carte, comme on le voit parfois, un signe textuel et une trace de terrain, attribuant à telle seigneurie, sur la foi d'une ou plusieurs mentions, la découverte et la transformation du minerai. Notre démarche tente d'éviter les faux-semblants du symbolisme : la carte que nous présentons est une carte de prospection où se dessinent cinq grands ensembles dont aucun n'échappe 
actuellement au recoupement de sources écrites, sans que pour autant les abondantes traces de terrain puissent encore être identifiées sur l'échelle du long terme.

Et si nous commencions par la fin de l'histoire? Tout le monde s'accorde pour écrire que la sidérurgie de la forêt d'Othe est défunte ou chancelante à l'extrême fin du $\mathrm{Xv}^{\mathrm{e}}$ siècle ou, selon les lieux, dans les premières décennies $d u \mathrm{xvI}^{\mathrm{e}}$ siècle. Il s'agit alors d'usines à fer, installées sur le cours des affluents de la Vanne elle-même, dont les sites demeurent dans le paysage actuel, et dont les bâtiments qui se sont succédé ont abrité diverses entreprises.

L'explication généralement avancée de ce déclin - l'épuisement des minières - n'est convaincante que si on l'assortit de conditions mortelles de concurrence: concurrence d'une nouvelle génération d'entreprises à une sidérurgie traditionnelle qui disparaît, concurrence entre "prédateurs", la demande de bois d'œuvre et de bois de chauffage assurant à une bonne gestion des forêts des revenus que la forge ne pouvait plus procurer, et entraînant la disparition précoce de quelques entreprises novatrices. Dans la mesure où la sidérurgie de la forêt d'Othe, touchée par un mouvement général en Europe, n'a pas survécu aux nouveautés, il vaut la peine de poser la question de l'innovation, même si les sources écrites sont, par définition, peu loquaces sur la fin des expériences, et, là comme ailleurs, avares de détails sur les débuts.

Reprenons donc, à la lumière des sources d'archives, l'histoire fragmentaire de la sidérurgie de vallée en forêt d'Othe à la fin du Moyen Âge. Le déplacement progressif, au $\mathrm{XIV}^{\mathrm{e}}$ siècle, de la sidérurgie des plateaux forestiers au réseau hydrographique de la Vanne et de ses affluents, Ancre, Nosle, ruisseau de Sévy, est attesté par la multiplication de forges et de moulins à émoudre, qui côtoient moulins à blé, à écorce, à chanvre ou à papier. Ces établissements sont accensés ou affermés dans l'aire de grands domaines princiers ou ecclésiastiques (évêché de Troyes, domaine ducal bourguignon, domaines cisterciens...) constitués autour de forts patrimoines forestiers; mais ils apparaissent aussi dans le cadre de la politique foncière de quelques seigneurs et officiers.

Comme partout, le vocabulaire traduit clairement ou suggère confusément les réalités techniques, et les progrès de l'innovation se lisent entre les lignes écrites par des administrateurs et des notaires. Le terme de "forge» est le plus courant, et le moins précis : on le rencontre aussi bien dans la métallurgie forestière (forge " Hube », forge du Mont-Erard) que sur le cours des rivières ; dans ce dernier cas, il désigne soit la forge de maréchal, soit la " grosse forge" créée à la fin du XIV siècle par la comtesse de Flandre, soit la forge d'affinerie d'Aix vers 1450, c'est-à-dire le second élément d'un établissement fonctionnant selon le procédé indirect. A cette date, un autre terme prend le relais et marque clairement la mise en place de la nouvelle technique, c'est celui de "fondoire ", attesté à Dilo en 1456 ( « saut à faire fondoire »), puis à Gerbeau en 1477; on décrit même les travaux de "dépeçage» de la "fondoire» de Craney en 1515-1516. Significatif de l'évolution, l'usage du terme « four ", que l'on rencontre à Maraye au xive siècle, à Craney en 1412, s'efface au profit du terme "fourneau ", comme en Normandie à la fin du $\mathrm{Xv}^{\mathrm{e}}$ siècle ${ }^{6}$, mais avec la précision embarassée qui atteste peut-être la nouveauté ou la rareté de ces structures : «fourneau de fondoire » ou «fourneau à faire le fer » dans l'aire de la seigneurie d'Aix-en-Othe au début du XVI ${ }^{e}$ siècle ; ateliers performants, comme l'indique l'importance des cens (20 à 25 livres tournois), mais qui seront, à Craney, à Chennegy, à Gerbeau, abandonnés par leurs exploitants et réduits en moulins à blé ou à papier. 
17 L'expérimentation du procédé indirect en forêt d'Othe s'inscrit dans un vaste mouvement qui touche, au cours $\mathrm{du} \mathrm{xv}^{\mathrm{e}}$ siècle, d'autres sites champenois (Rimaucourt, régions de Saint-Dizier et de Joinville), mais aussi des zones limitrophes de la Champagne: Bourgogne (Bèze, Renève), Franche-Comté (Bley, vallée de la Romaine), Puisaye (Les Andressis). Peut-on remonter à ces origines ?

18 Le terrier de la seigneurie d'Aix-en-Othe, dressé au milieu du $\mathrm{XV}^{e}$ siècle, précise que la " forge à faire le fer ", dite aussi " marteau et affinière ", installée sur le cours de la Nosle au-desus d'un moulin à " esmoudre ferrements » et d'un moulin à chanvre et écorce, est en ruine, et «ne sest riens passés de V a VI ans ». On n'a pas retrouvé d'autres traces explicites de cette installation dans la comptabilité épiscopale, qui présente quelques lacunes au milieu du $\mathrm{Xv}^{\mathrm{e}}$ siècle. Mais sur un autre site de la vallée de la Vanne, on peut remonter près de 80 ans en arrière pour rencontrer le premier témoignage d'adoption du procédé indirect dans la région, contemporain d'exemples connus pour le Berry et le Val de Saône : il s'agit de la forge de Chicheré à Saint-Liébaut.

C'est dans les années 1370 que Nicolas de Fontenay, seigneur de Saint-Liébaut, bailli de Troyes et grand officier ducal, succédant à Saladin d'Anglure, reprend de Guy de Pontailler, maréchal de Bourgogne, lui-même engagé dans la métallurgie en Val de Saône, les terres de Chennegy et de Valcon. En 1377, Nicolas de Fontenay prend à ferme pour dix ans des religieux de Dilo leur moulin de Chicheré, où il devait faire «deux moulins, a savoir un moulin a draps par devers Loigny et un autre boutoir a ecorce du coste par devers Saint Liebaut ». Au bout des dix ans, les religieux constatent que rien de ce qui avait été prévu n'avait été réalisé, et que leur ancien moulin était bien en ruine. En réalité, dès la conclusion du bail, le fermier avait aménagé la rivière, fait construire « une forge a affiner fer » munie d'« une grant roe a faire soufler grans souflez», dont un certain Collesson le Liégeois prenait la direction. Nicolas de Fontenay prenait en 1378 à cens la traite du minerai des terres de la seigneurie d'Aix et, en 1380, acquérait de Saladin d'Anglure la terre de Chasoy, près de Villemaur, le grand étang de Valcon, un moulin et 400 arpents de bois. Il s'assurait en somme le minerai et le combustible nécessaires à l'entreprise.

D'abord réticents à s'attaquer à un homme puissant, les religieux obtinrent justice après la mort de Fontenay : un arrêt du roi ordonna la destruction de la grande roue, la remise en état du site avec deux moulins à écorce et à draps, et imposa aux héritiers 120 écus d'or de dédommagement. C'est à la plus jeune des filles qu'était échue, entre autres biens à Chennegy et Valcon, la « forge à l'eau » de Saint-Liébaut, qui devint un lieu-dit : en 1429, on évoquait toujours le "gué des forges ». Mais l'usine à fer s'était déplacée : un siècle plus tard, les seigneurs de Saint-Liébaut conservent un ensemble cohérent dans la vallée de l'Ancre. Une forge existe à Valcon, on y vend en 1519 deux cents et demi de fer; en 1520 , un «saut et place ou d'ancienneté avoit une fondoire a fer seant a Chennegy » est pris à rente. On voit alors apparaitre une famille de marchands de Troyes, installée à Paris, les Boucherat, qui ont des intérêts à Valcon et afferment un temps le « fourneau à fer » d'Aix ; mais ce sont les derniers feux champenois à l'Ouest de Troyes. Seule l'usine de Vandeuvre-sur-Barse demeure après 1520 susceptible d'approvisionner le marché régional en fontes.

21 Hors des vallées où nous a entraînés l'enquête sur la fin d'une histoire millénaire, cinq à six grands ensembles miniers et métallurgiques apparaissent sur la carte, tels qu'ils se dessinent dans notre documentation. Des dossiers particulièrement riches relatifs à la seigneurie épiscopale d'Aix éclairent au cœur du massif boisé des entreprises 
contemporaines des grosses forges de la comtesse de Flandre ; c'est sur ces sources que nous concentrerons ici l'analyse, en insistant sur les sites et en remettant à une présentation ultérieure le tableau qu'elles offrent sur une micro-société en pays de forêt à la fin du XIv ${ }^{\mathrm{e}}$ siècle.

Parmi les terres relevant de la seigneurie épiscopale d'Aix figure le Mont-Erard. Plusieurs indices concordants nous permettent aujourd'hui d'identifier ce toponyme disparu avec l'actuel hameau du Mineroy: des confins mentionnés dans notre documentation: «les laies et séparations des bois de Nullon et de Jarruyer tirent droit au Mont-Erard... » ou les " cornées » du Mont-Erard, qui correspondent aux hauteurs culminant à $232 \mathrm{~m}$, des Cornées Laliat, Alexandre et Cabourdin sur le territoire de la commune d'Aix-en-Othe ${ }^{7}$.

Vers 1370, il s'agit d'un vaste chantier, dont l'administration avait été confiée à un "gouverneur des forges et forêts ", et qui ne peut être dissocié de l'hôtel de l'évêque à Aix-en-Othe, centre de gestion et de stockage des produits finis. C'est d'abord un domaine boisé. L'évêque exploite son droit de panage, vend le produit de ses coupes, bois de chauffe, merrain à bâtir, échalas pour les vignes, lattes, ais et aisselles.

La présence de minerai de fer engendre d'autre part une activité métallurgique intense et des revenus appréciables, supérieurs à ceux que l'on retire de l'exploitation du bois. Le fer est réduit sur place et se présente en sommes, comme en témoigne l'inventaire de lieux dressé en 1370, à la mort de l'évêque Henri de Poitiers.

A partir de cette date, la régie directe semble abandonnée, bois et minerais sont dissociés, une recomposition économique et sociale du paysage forestier s'opère au bénéfice d'exploitants et de marchands extérieurs au lieu et étrangers au milieu de colons, mineurs et bûcherons installés par l'évêque en lisière des bois. On voit apparaître ou réapparaître de nouveaux acteurs, les habitants de la vallée de la Nosle qui affirment leur droit d'usage et peuvent vendre leur minerai, "si l'évêque n'y fait miner pour lui et en son nom ", des entrepreneurs, comme Nicolas de Fontenay évoqué plus haut, qui prend à bail la traite des minerais du Mont-Erard en 1378-1379, ou des marchands de fer, souvent associés en famille, comme les Chevrillon, dont on retrouve la trace comme amodiateurs de forges ou de moulins à émoudre ; les ventes de bois se succèdent, bois des Cornées de Mont-Erard acheté en 1379-1380 par Jehannin Chappelain, 50 arpents achetés en 1382-1383 par Pierre Breton et Felix Jehannel, ce dernier exploitant alors la forge de Surançon, 50 arpents encore en 1384-1385 par les héritiers de Jehannel ; changements de main, glissement vers la vallée, cependant que les recettes de forestage, qui connaissent leur maximum vers 1385-1390, régressent jusqu'en 1430, puis disparaissent des registres.

L'enquête de terrain dans une zone où les traces d'activité métallurgique sont particulièrement denses, permettra peut-être de confirmer les limites chronologiques que suggère la séquence des mentions relatives à l'exploitation des minières. Sinon, comment situer des indications de revenus ou de transactions dans un espace plus large et un réseau de relations qui nous échappe ? Deux autres exemples, tirés des sources écrites, illustrent les difficultés de l'enquête et l'utilité méthodologique des sondages projetés.

Transportons-nous d'abord sur le territoire de Pâlis, sur la rive droite de la Vanne, d'où provenait le minerai approvisionnant, par charroi, la forge de Villemaur, mais aussi les forges de Maraye et de Surançon vers 1370. La moitié de la terre et justice de Pâlis appartenait à la fin du XIV ${ }^{e}$ siècle à la famille de Broutières. En 1371, l'écuyer Jacques de Broutières perçoit 40 sous pour revenu du minerai, soit l'équivalent du revenu de la 
justice haute, moyenne et basse de cette seigneurie et quatre fois moins que le revenu des tailles. Or, on ne trouve pas mention du revenu du minerai dans deux autres dénombrements du fief, celui de 1361 rendu par Jehan de Dinteville à la comtesse de Flandre, et celui de 1393 rendu par Jehan de Broutières au duc de Bourgogne; ces deux dates circonscrivent dans le temps ce que l'on sait de l'extraction du minerai de fer en rapport avec l'activité de trois grosses forges : n'y aurait-il extraction qu'en fonction de débouchés propres à la seigneurie ?8

Autre exemple, qui nous ramène dans les confins du Mont-Erard, disputés entre l'évêque de Troyes et l'abbé de Pontigny. Une enquête effectuée en 1539 et rapportée à la fois dans les fonds d'archives des deux parties rappelle le cours d'une affaire vieille de trois siècles, plusieurs fois réglée, et qui avait dégénéré dans le premier tiers $\mathrm{du} \mathrm{xvI}^{\mathrm{e}}$ siècle (emprisonnement de marchands cendriers et saisie de leurs biens par l'abbé considérant qu'ils travaillaient indûment sur ses terres). Dans ces confins, tels qu'on les saisit au terme d'une longue histoire, les bois étaient l'objet d'une intense activité : façon de cendres pour le compte de marchands de Sens et de Paris, façon de charbon, fendage de bois, façon de cercles, de merrain, de lattis; un témoin rapporte que des porchers se fournissaient chez son père en sel, huile, eau, fer et autres denrées. Au début $d u \mathrm{XVI}^{\mathrm{e}}$ siècle, on continue d'extraire du minerai des bois de Jarruyer, mais on ne le réduit plus sur place et il est charroyé vers les sites de vallées évoqués plus haut, tel celui de la forge de Cosdon sur la Vanne. Mais la comptabilité épiscopale permet de suivre une importante opération de défrichement, avec l'installation de colons et la mise en labours de 415 arpents, soit plus de 200 hectares de terres situées sur d'anciennes zones d'extraction et de réduction du minerai de fer: désormais, le site cesse de s'appeler la "Vallée aux Poëles » pour prendre le nom de « Grande Vallée ».

L'enquête de 1539 présente un premier intérêt qui est de signaler les limites administratives entre deux seigneuries dans une zone où la vivacité des querelles de bornage tenait à la qualité des ressources, en particulier à la présence des minerais de fer.

Le second intérêt est, à travers les témoignages, archéologique : les témoins font appel à leur mémoire, et rien n'indique, dans leur description des lieux, qu'ils aient vu mines et forges en activité : l'un d'entre eux se souvient qu'il y a 17 ans, on a planté une borne près $\mathrm{du}$ « ferry au Nain, ferrier de grands et gros monceaux d'écume de fer »; mais le site de référence était déjà bien mort, comme tous les autres sites décrits par les rejets de fours et non par les installations. L'expression «le lieu qu'on appelle les ferriers» est, de ce point de vue, significative. Comme des divergences apparaissent entre témoins sur la dénomination de tel ou tel ferrier, on peut en conclure que la mémoire métallurgique des lieux est encore très forte, mais qu'il faut remonter à une génération pour dater les derniers temps des fours forestiers et des forges qui les accompagnaient (vallée des Poëles) : on pourrait même remonter plus haut, si un premier sondage dans l'un des ferriers de la zone n'avait fait surgir un indice de datation de la fin du Moyen Age.

31 Pour d'autres sites de la forêt d'Othe ou pour des époques antérieures, on ne manque pas de témoignages comparables, liés soit à des problèmes de bornage, soit à des controverses sur les usages. Il s'agit, par exemple, d'une enquête sur les biens et droits de l'abbaye de Vauluisant en 1503-1504, au long des chemins séparant les bois de Rajeuses des bois de la verrerie de Fournaudin, où " $\mathrm{y}$ a grand foison de mynières ", ou du bornage entre Cérilly et Rigny-le-Ferron, «ensuivant les placquis et marques faites au marteau et coin des religieux jusqu'à... l'endroit d'un charme marqué assis sur un ferrier près dudit chemin ». On dispose de compromis, parfois très détaillés, entre seigneurs, comme les sires de 
Brienne ou de Venizy, et des religieux, comme les moines de Dilo, de Pontigny et de Vauluisant, qui aident à préciser la topographie des zones d'extraction et de transformation aux XIII ${ }^{e}$ et $\mathrm{XIV}^{\mathrm{e}}$ siècles, ainsi une longue description des chemins entre Mont-Erard et Berluvier, où sont évoqués non plus des ferriers mais des fours et des forges en activité9.

Parvenus au point où s'amorce, sur quelques ensembles miniers et métallurgiques, la conjonction entre sources d'archives et traces archéologiques, nous abordons une phase nouvelle de l'enquête. Nous n'espérons plus découvrir de nouvelles sources comptables, qui permettent d'évaluer la capacité productive du pays d'Othe, même pour la fin du XIV siècle, pour laquelle nous disposons de quelques chiffres. Dans les zones où les ferriers n'ont pas fait l'objet de prélèvements massifs, la prospection systématique va nous offrir des possibilités de calcul à partir de la masse des scories de réduction; encore faut-il que les indices chronologiques précisent l'identité et la durée des ateliers, individualisent des phases d'activité.

Dans plusieurs secteurs, les sources ne manquent pas - ni les travaux préliminaires ${ }^{10}$ - pour reconstruire l'histoire de la gestion des espaces forestiers: les bois de Pâlis, les forêts épiscopales, les bois des seigneuries laïques et monastiques autour de Séant (Bérulle), Bœurs et Dilo ont fait l'objet d'une exploitation systématique, dont la sidérurgie représente l'un des enjeux visibles ou détectables.

Ayant tenté de prendre la mesure du fait industriel et des mutations techniques qui provoquent déclins et déplacements, nous avons l'espoir de faire revivre les acteurs: communautés rurales, seigneurs et marchands. A travers la politique des individus et des groupes, les niveaux de l'entreprise, les calculs de l'engagement novateur ou la gestion au jour le jour, c'est la place de la forêt d'Othe dans l'économie régionale, mais aussi dans l'histoire de la sidérurgie européenne qui pourrait être précisée. C'est sur ces objectifs que se clôt ce bilan.

\section{NOTES}

1. D. Cailleaux, «Les religieux et le travail du fer en pays d'Othe", in Moines et métallurgie dans la France médiévale, études réunies par P. Benoit et D. Cailleaux, Paris, 1991, pp. 193-211, a le mérite de présenter sur une carte l'essentiel des données topographiques rassemblées à partir des travaux de T. Boutiot, A. Huré, M. Quantin. Parmi les publications essentielles: A. Longnon, Documents relatifs au comté de Champagne et de Brie (1172-1361), I- Les fiefs, Paris, 1877 ; II- Le domaine comtal, Paris, 1904 ; A. Huré, «Origine et formation du fer dans le Sénonais. Ses exploitations et ses fonderies dans l'Yonne », Bulletin de la Société des Sciences historiques et naturelles de l'Yonne, 73, 1919, pp.33-106, qui n'aborde la forêt que dans sa partie occidentale; M. Quantin, Cartulaire général de l'Yonne, t. I et II, Auxerre, 1854 et 1860, et Recueil de pièces pour faire suite au cartulaire général de l'Yonne, Auxerre, 1883 ; A. Roserot, Dictionnaire historique de la Champagne méridionale des origines à 1790. Langres, 1945.

2. Ph. Braunstein, «Les forges champenoises de la comtesse de Flandre (1372-1404) », Annales E.S.C., 1987, pp. 747-777. 
3. P. Beck, Ph. Braunstein, C. Dunikovski, M. Philippe, «La sidérurgie ancienne en forêt d'Othe, in Proto-industries et histoire des forêts, Actes du colloque interdisciplinaire de l'an 1990, sous presse.

4. Nous remercions le C.R.H. qui a contribué au financement de cette enquête inscrite à son programme et le C.I.H.A.M. de Lyon, pour son aide logistique.

5. Le cubage est calculé par la formule des segments sphériques; le tonnage, d'après l'indice proposé par G.Sperl, Uber die Typologie urzeitlicher, frühgeschichtlicher und mittelalterlicher Eisenhüttenschlaken, Vienne, 1980. Le constat est comparable à celui que formulait pour le Morvan M. Mangin, "Les mines et la métallurgie du fer en Gaule romaine: travaux et recherches ", Latomus, XLVII, 1988, p. 82 : « ferriers d'importance modeste, entre 100 et $1000 \mathrm{~m}^{3}$... »

6. La métallurgie normande (XII $\mathrm{e}-\mathrm{XVII}{ }^{\mathrm{e}}$ siècles). La révolution du haut-fourneau, par J.F. Belhoste, Y. Lecherbonnier, M. Arnoux, D. Arribet, B.G. Awty, M. Rioult, Cahiers de l'Inventaire, $\mathrm{n}^{\circ} 14$, Caen, 1991, p. 47.

7. Le Mont-Érard ne se situe donc pas au lieu-dit Mont Saint-Benoît, mais sur l'autre côté de la vallée de la Nosle ; cf. Ph. Braunstein, Les Forges..., p. 757.

8. De ce rapport entre les ressources naturelles et l'instrument de transformation, on trouve une définition contractuelle dans la transaction entre le chevalier de Foissy et les religieux de Vauluisant qui ne reçoivent des mines de fer dans les bois qu'autant qu'un fourneau peut souffler. A.D. Yonne, H 724 (1198) ; cf. M. Quantin, Cartulaire général..., 2, p. 488.

9. Cartulaire de Pontigny, fo 294 , B.N., Ms. lat. 5465.

10. Par exemple, H. Rübner, Untersuchungen zur Forstverfassung des mittelalterlichen Frankreichs, Vierteljahrschrift für Sozial und Wirtschaftsgeschichte, Beiheft 49, qui propose une carte de la gruerie de Villemaur en 1328.

\section{AUTEURS}

\section{PATRICE BECK}

Patrice Beck est maître de conférences à l'Université de Tours.

\section{PHILIPPE BRAUNSTEIN}

Philippe Braunstein est directeur d'études à l'EHESS et membre du Centre de Recherches Historiques.

\section{MICHEL PHILIPPE}

Michel Philippe est chercheur à l'Inventaire (cellule du Patrimoine Industriel). 